\title{
Review: wound cleansing with water does not differ from no cleansing or cleansing with other solutions for rates of wound infection or healing
}

Fernandez R, Griffiths R, Ussia C. Water for wound cleansing. Cochrane Database Syst Rev 2002;(4):CD003861 (latest version 19 Oct 2001).

\begin{abstract}
QUESTION: In patients with acute or chronic wounds, is wound cleansing with water (tap, cool boiled, or distilled) more effective than no cleansing, or wound cleansing with sterile normal saline or any other solution for reducing infection and improving healing?
\end{abstract}

\section{Data sources}

Studies were identified by searching the Cochrane Wounds Group Specialised Trials Register, Medline, EMBASE/Excerpta Medica, CINAHL, and the Cochrane Controlled Trials Register. Bibliographies of relevant articles were reviewed. Primary authors, experts, and company representatives in the field of wound care were contacted for further studies.

\section{Study selection}

Studies published in any language were selected if they were randomised controlled trials (RCTs) or quasirandomised controlled trials (QRCTs) comparing wound cleansing using water (tap, cool boiled, or distilled) with no cleansing or wound cleansing using sterile normal saline or any other solution in patients of all ages with wounds of any aetiology, in any setting (hospital, community, nursing home, general practice, or wound clinic).

\section{Data extraction}

Data were extracted independently by 2 reviewers on patient and wound characteristics, details of the intervention, tap water quality, concurrent interventions, study setting, duration of follow up, number and reasons of withdrawals, and outcomes. Outcomes included rates of wound infection and wound healing.

\section{Main results}

2 RCTs and 4 QRCTs (with follow up ranging from 1-6 wks) met the selection criteria. 2 trials compared wounds cleansed using tap water with those not cleansed, and 4 trials compared wound cleansing using water with other solutions. (1) Tap water $v$ no cleansing. Meta-analysis of 2 QRCTs showed that the groups did not differ for rate of infection (pooled odds ratio [OR] 1.06, 95\% CI 0.06 to 17.47). 1 of the trials reported that the groups did not differ for rate of wound breakdown (OR 0.57, CI 0.04 to 9.17). (2) Tap water $v$ normal saline (1 RCT and 1 QRCT). The QRCT reported a lower infection rate in wounds (acute soft tissue wounds that were sutured) cleansed with tap water than those cleansed with normal saline (relative risk 0.55 , CI 0.31 to 0.97 ). The RCT reported that the groups did not differ for wound infection (OR 0.14 , CI 0.01 to 2.92) or wound healing (OR 0.33, CI 0.10 to 1.07). (3) Distilled water and/or cool boiled water $v$ normal saline (1 RCT). When results for distilled and cool boiled water were pooled and compared with isotonic saline, the groups did not differ for number of infections
(OR 0.55, CI 0.18 to 1.62). (4) Tap water v procaine spirit (1 QRCT). The groups did not differ for number of infections, and by day 14 of follow up, all the wounds had healed well.

\section{Conclusion}

In patients with acute or chronic wounds, wound cleansing with water does not differ from no cleansing, or wound cleansing with sterile normal saline or any other solution for rates of healing and infection.

\section{COMMENTARY}

Appropriate wound cleansing continues to be debated in wound management circles. Isotonic saline and more recently, tap water, are most commonly recommended. Limited research has been available to support or refute the use of these solutions. The review by Fernandez et al provides some clarity about the comparative benefits of these cleansing solutions in wound care.

The central clinical questions relate to the relative merits of tap water versus sterile saline for wound cleansing, and whether patients with surgical wounds should shower with their wounds exposed. Water quality may influence outcomes, and populations living in places where the quality of tap water is poorer than in the studies in this review may have different outcomes. The finding that distilled water and boiled water do not increase infection rates compared with saline suggests that areas with poor quality tap water can employ measures to make it safe. As the studies did not include children, the authors recommend not using tap water to clean children's wounds. However, a recently published RCT compared tap water with saline for clean lacerations in children 2-18 years of age and found that infection rates did not differ between the 2 groups. ${ }^{1}$ Of particular relevance to surgical areas, is the support the review lends to the practice of patients with closed surgical wounds showering with their wounds exposed. This may help to resolve current inconsistencies in this practice.

The results of the review suggest that tap water is safe to use as a cleansing solution for wounds. Because the studies were done in hospital wards, emergency departments, and community settings, the results are broadly applicable. However, the decision to use tap water as a cleansing solution for wounds should take into consideration the quality of tap water, comorbidities that compromise immune function of the patient, and the extent and nature of the wound.

Julie Betts, RGON, AdDipN Resource Nurse, Wound Management Health Waikato Hamilton, New Zealand

1 Bansal BC, Wiebe RA, Perkins SD, et al. Tap water for irrigation of lacerations. Am J Emerg Med 2002;20:469-72.
Source of funding: no external funding.

For correspondence: Ms R Fernandez, Centre for Applied Nursing Research, Liverpool BC, New South Wales, Australia. ritin.fernandez@swsahs.nsw.gov.az 